\title{
Estudio de incidencia de riesgos y eventos vinculados a la seguridad en una unidad de cuidados intensivos
}

\author{
Dres. Laureano Leyes*, Fausto Porcires ${ }^{*}$, Mario Godino*, Marcelo Barbato ${ }^{\dagger}$

\section{Resumen}

Introducción: los pacientes críticos son muy vulnerables a la iatrogenia. Los eventos vinculados a la seguridad (EVS) contribuyen en la morbilidad y mortalidad de los pacientes ingresados. Por esto, su detección y prevención son importantes.

Objetivos: estudiar la incidencia de EVS seleccionados en una unidad de cuidados intensivos (UCI) y el daño que generan. Sentar las bases para un sistema de notificación de EVS.

Metodología: estudio prospectivo de incidencia mediante dos metodologías: reporte voluntario y auditoría de historias clínicas.

Resultados: incluyó 174 pacientes. Se detectaron 107 EVS en el 35\% de los pacientes. Los más prevalentes fueron la salida de sondas y catéteres $(42 \%)$ y los relacionados a medicación de alto riesgo $(12,8 \%)$. La edad $(p=0,046)$, tiempo de estancia en $\mathrm{UCl}(p=0,001)$ y requerimiento de sedoanalgesia $(p=0,039)$ se asociaron en forma independiente a la presencia de EVS. El $76,6 \%$ de los eventos generó daño, pero no se asoció a mayor mortalidad $(p=0,96)$.

Conclusiones: los EVS son altamente frecuentes en UCI. La salida de sondas y catéteres son los más prevalentes. Se asocia con el requerimiento de asistencia ventilatoria, la sedoanalgesia y la estancia en $\mathrm{UCl}$. El reporte voluntario es una herramienta útil para su detección.

Palabras clave: Seguridad del paciente

Unidades de cuidados intensivos

Evento adverso

Daño del paciente

Key words: $\quad$ Patient safety

Intensive care units

Adverse event

Patient harm

\footnotetext{
* Médico intensivista.

† Jefe de Servicio.

Unidad de Cuidados Intensivos. Hospital Maciel.

Correspondencia: Dr. Laureano Leyes. Correo electrónico: laurus022@hotmail.com.uy

Recibido: $28 / 6 / 19$

Aprobado: $7 / 10 / 19$
} 


\section{Introducción}

El error es una condición inherente a toda actividad humana. La medicina no escapa de esta noción y durante su práctica el error genera daño sobre los pacientes. Los estudios que evalúan la presencia de errores durante la internación hospitalaria existen desde 1964, cuando se determinó que hasta $20 \%$ de los pacientes ingresados sufren lesiones iatrogénicas vinculadas a errores ${ }^{(1)}$. Desde ese momento, el número de estudios que encaran esta temática ha aumentado drásticamente. En alguno de ellos la prevalencia de eventos iatrogénicos se detectó hasta en 36\% de los pacientes, dentro de los cuales el $25 \%$ amenazó la vida de éstos ${ }^{(2)}$. En un estudio realizado en Harvard con más de 30.000 pacientes los eventos adversos se presentaron en el $4 \%$ de estos, generando en el $75 \%$ de los casos algún tipo de discapacidad y en $14 \%$ la muerte ${ }^{(3)}$.

Sin embargo, desde estos estudios, la práctica de la medicina ha cambiado en forma desmedida, agregando nuevas tecnologías y adoptando técnicas más invasivas. En 1999, Chantler afirmó que "antes la medicina era sencilla, poco efectiva y relativamente segura; hoy es compleja, efectiva y potencialmente peligrosa"(4). En gran parte por estos motivos, los pacientes críticos son muy vulnerables a la iatrogenia.

Para reducir la frecuencia de los problemas de seguridad es necesario entender sus causas y diseñar métodos para prevenirlos o detectarlos antes de que produzcan daño a los pacientes. De ahí que aprender de los propios errores se ha convertido en uno de los objetivos de las estrategias para la seguridad de los pacientes. Una forma de conseguir esto es tener un sistema de registro y notificación de incidentes y eventos adversos que permita su identificación para posteriormente crear estrategias para su reducción. Conocer con detalle la cadena de acontecimientos que culmina con un incidente para la seguridad del paciente, representa una oportunidad para prevenir posibles ocurrencias de situaciones similares en otros pacientes $y$, por tanto, debiera contribuir a reducir los eventos adversos ${ }^{(5)}$.

Teniendo en cuenta todo lo antedicho, podemos decir que los eventos adversos contribuyen en la morbilidad y mortalidad de los pacientes ingresados y mucho de ellos son prevenibles. Por esto, su identificación y la implementación de medidas para disminuir su prevalencia son de vital importancia.

\section{Objetivos}

- Estudiar la incidencia de eventos vinculados a la seguridad seleccionados en una UCI polivalente.

- Valorar el daño que producen y su severidad.
- Sentar las bases para un sistema de notificación de estos eventos.

\section{Material y método}

Estudio prospectivo observacional.

\section{Población estudiada}

Se realizó en el Hospital Maciel de la ciudad de Montevideo, Uruguay. Constituye una unidad de cuidados intensivos polivalentes que cuenta con 19 camas de UCI y 5 camas de cuidados intermedios.

Incluye todos los pacientes ingresados a UCI en un período de dos meses (25/8/14 a 25/10/14). Se analizaron variables cuantitativas como edad, estadía en la UCI y SAPS II, y cualitativas como sexo, motivo de ingreso, procedencia, reingreso, requerimiento de asistencia respiratoria mecánica (ARM), vasopresores y mortalidad en la unidad. Los datos fueron extraídos de las historias clínicas.

\section{Eventos vinculados a la seguridad}

Se define evento vinculado a la seguridad (EVS) como aquel que puede generar un daño innecesario, el cual es imputable a la atención de salud y no está relacionado con la enfermedad que padece el paciente. Cuando este evento genera daño se denomina evento adverso, de lo contrario constituye un incidente vinculado a la seguridad.

Se seleccionaron 16 eventos predefinidos a estudiar, los cuales se detallan en la tabla 1. Fueron seleccionados por el equipo de calidad y seguridad del servicio (del que los autores son parte) con base en estudios internacionales, como los eventos con mayor impacto sobre nuestros pacientes, ya sea por su alta frecuencia o gravedad.

Se realizó previo al inicio del estudio un período de difusión que incluyó charlas informativas al personal del servicio y envío de la información pertinente vía correo electrónico.

El estudio de incidencia se realizó mediante la aplicación de dos metodologías:

1. Reporte voluntario de eventos mediante herramienta de captura en formato papel diseñada para tal fin. Fue anónima y realizada por los diferentes miembros del equipo de salud (médicos residentes, médicos intensivistas, licenciadas de Enfermería, enfermeras). En la misma se detalla tipo de evento, quién lo reporta, el turno en el que ocurre, así como la unidad para posterior identificación del paciente. En cada sector de la unidad se colocaron planillas para el reporte, buzones cerrados donde depositarlas y cartelería con las definiciones de los incidentes seleccionados. 
Tabla 1. Eventos vinculados a la seguridad seleccionados.

$\begin{array}{ll}\text { Salida incidental de sondas, catéteres y drenajes } & \text { Retraso en TQT quirúrgica (mayor de } 5 \text { días) } \\ \text { Neumotórax por vía } & \text { Falla de equipamiento } \\ \text { Obstrucción de SOT o TQT } & \text { Retraso en diagnóstico microbiológico } \\ \text { Errores asociados a medicación de alto riesgo (MAR)* } & \text { Alta de UCI después de la hora } 22 \\ \text { Infección asociada a catéter } & \text { Úlceras por presión (UPP) } \\ \text { Retraso en tratamiento quirúrgico } & \text { Extravío de muestra biológica } \\ \text { Meningitis o ventriculitis posneuroquirúrgicas } & \text { Caída de cama o camilla } \\ \text { Retraso en imagen de urgencia (mayor a 2 horas) } & \text { Paro cardiorrespiratorio durante traslado }\end{array}$

* Medicamentos que tienen un riesgo muy elevado de causar daños graves o incluso mortales cuando se produce un error en el curso de su utilización.

Tabla 2. Clasificación de los errores según su gravedad

\begin{tabular}{|c|c|}
\hline $\begin{array}{l}\text { Categoría de } \\
\text { gravedad }\end{array}$ & Definición NCC MERP(17) \\
\hline Categoría A & $\begin{array}{l}\text { Circunstancias o incidentes con capacidad de causar } \\
\text { error }\end{array}$ \\
\hline Categoría B & El error se produjo, pero no alcanzó al paciente \\
\hline Categoría C & El error alcanzó al paciente, pero no le causó daño \\
\hline Categoría D & $\begin{array}{l}\text { El error alcanzó al paciente y no le causó daño, pero } \\
\text { precisó seguimiento para comprobarlo }\end{array}$ \\
\hline Categoría E & $\begin{array}{l}\text { El error contribuyó o causó daño temporal al paciente } \\
\text { y precisó intervención }\end{array}$ \\
\hline Categoría F & $\begin{array}{l}\text { El error contribuyó o causó daño temporal y precisó o } \\
\text { prolongó la hospitalización }\end{array}$ \\
\hline Categoría G & $\begin{array}{l}\text { El error contribuyó o causó daño permanente al } \\
\text { paciente }\end{array}$ \\
\hline Categoría H & El error afectó la vida del paciente \\
\hline Categoría I & El error contribuyó o causó la muerte del paciente \\
\hline
\end{tabular}

2. Auditoría de historias clínicas de UCI por parte de los autores. No se analizaron circunstancias de daño potencial en otros sectores del hospital, ya sea previo o posterior al alta de UCI.

Posteriormente, se realizó el análisis de incidencia mediante la conjunción de ambos métodos mencionados.

Los diferentes eventos se clasificaron según la presencia y severidad del daño mediante el índice para la categorización de errores de National Coordinating

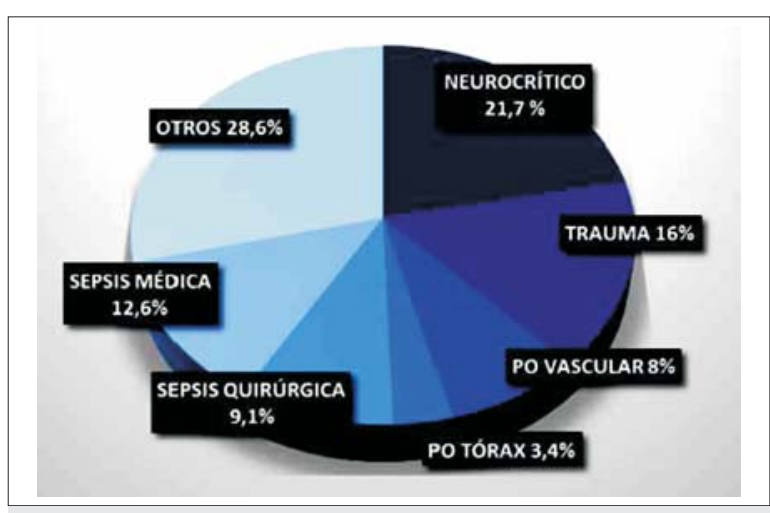

Figura 1. Motivo de ingreso a UCl.

Council for Medication Error Reporting and Prevention $(\text { NCC MERP) })^{(6)}$. Si bien esta clasificación fue desarrollada para categorizar los errores vinculados a la medicación, estas definiciones se pueden aplicar a cualquier tipo de error o evento adverso (tabla 2). En dicha clasificación las categorías A y B constituyen episodios de riesgo de sufrir eventos adversos; $\mathrm{C}$ y D incidentes sin daño, y los restantes eventos adversos con daño de diferente severidad.

\section{Análisis estadístico}

Los análisis estadísticos se realizaron utilizando el programa SPSS Statistics. Las distintas variables continuas se expresaron como media y su desvío estándar. Para comparar proporciones con variables nominales se utilizó el test de chi cuadrado y para frecuencias esperadas pequeñas se empleó el test exacto de Fisher. Se consideró estadísticamente significativo un valor de $\mathrm{p}<0,05$.

Para el análisis multivariado se incluyeron aquellas variables con una $\mathrm{p}<0,2$ y se utilizó un test de regresión logística bimodal. Se registró el OR \pm IC $(5 \%$ - 95\%) y 
Tabla 3. Comparación de métodos para la detección de los diferentes eventos vinculados a la seguridad y frecuencia de éstos (proporción del total de eventos registrados).

\begin{tabular}{|c|c|c|c|c|c|}
\hline & $\%$ & Auditoría & Reporte & Total & $p$ \\
\hline MAR & 12,8 & 5 & 10 & 13 & ns \\
\hline Bacteriemia por catéter & 3,9 & 4 & 0 & 4 & 0,014 \\
\hline Retraso imagen & 8,8 & 9 & 0 & 9 & $<0,001$ \\
\hline Retraso TQT & 6,9 & 4 & 3 & 7 & ns \\
\hline Hora alta & 6,9 & 7 & 0 & 7 & $<0,001$ \\
\hline UPP & 9,8 & 10 & 2 & 10 & $<0,001$ \\
\hline Caídas & 2,0 & 2 & 0 & 2 & ns \\
\hline Salida sondas, catéteres & 42,2 & 30 & 23 & 43 & ns \\
\hline Extravío muestra & 3,9 & 2 & 4 & 4 & ns \\
\hline Falla de equipo & 1,0 & 1 & 0 & 1 & ns \\
\hline \multirow[t]{2}{*}{ Neumotórax por procedimiento } & 6,9 & 6 & 5 & 7 & ns \\
\hline & $100 \%$ & $80(75 \%)$ & $47(44 \%)$ & 107 & \\
\hline
\end{tabular}

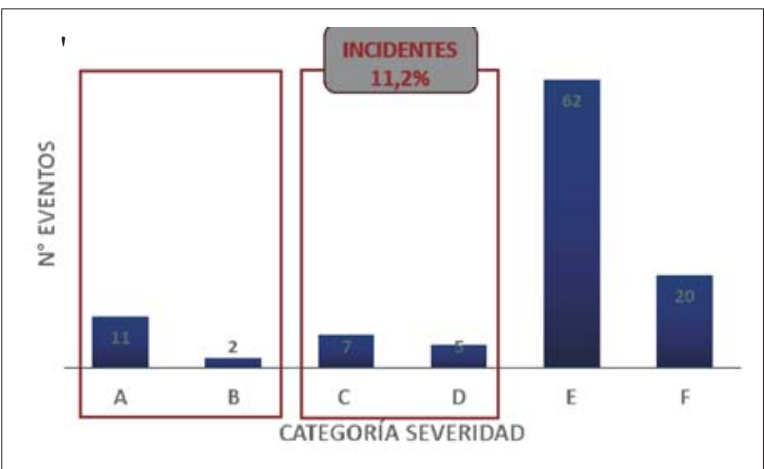

Figura 2. Severidad de eventos asociados a seguridad.

se consideró estadísticamente significativo un valor de $\mathrm{p}<0,05$.

\section{Resultados}

\section{Características de la población}

En el período analizado ingresaron 174 pacientes que fueron incluidos en el trabajo.

El $60 \%$ de la población era de sexo masculino, con una edad media de 54,8 \pm 20 años. El 58\% requirió AVM y el 39\% apoyo vasopresor, con un SAPS II al ingreso de $36 \pm 23$ y una estadía en la unidad de $9 \pm 11$ días. La mortalidad en la unidad para la población estudiada fue de $27,7 \%$.

La patología neurológica crítica y la sepsis fueron las causas de ingreso más frecuentes. La frecuencia de los diferentes motivos de ingreso se muestra en la figura 1 .

\section{Frecuencia de eventos vinculados a la seguridad}

Se auditaron 166 historias clínicas (95,4\% del total), dada la imposibilidad de conseguir la documentación restante por dificultades administrativas. Concomitantemente se recibieron 51 reportes de eventos, cuatro de los cuales $(7,8 \%)$ debieron descartarse, ya que no correspondían a pacientes incluidos en el estudio. Por último, se compararon los resultados eliminando las coincidencias. De este análisis se extrajeron los resultados expuestos a continuación.

Se detectaron un total de 107 eventos durante el período analizado.

El 35\% de los pacientes presentó algún evento vinculado a la seguridad $(22,4 \%$, uno; $5,7 \%$, dos; $6,9 \%$ más de dos eventos).

La salida de sondas, catéteres y drenajes fue el evento más frecuente, constituyendo el $42,2 \%(n=30)$ del total. Dentro de éstos, las extubaciones no programadas constituyeron el $40 \%(n=12)$. Los eventos asociados a medicación de alto riesgo (MAR) le siguieron en frecuencia constituyendo el $12,8 \%$ del total de eventos detectados $(\mathrm{n}=13)$.

No se detectaron PCR durante el traslado, meningitis ni ventriculitis posneuroquirúrgicas, retrasos en tratamiento quirúrgico y obstrucción de sondas orotraqueales (SOT) o de traqueostomías (TQT).

La frecuencia del resto de los eventos seleccionados se muestra en la tabla 3.

\section{Comparación de métodos para la detección de eventos} vinculados a la seguridad

De los 107 eventos recuperados la auditoría de historias clínicas detectó $80(74,7 \%)$. 
Tabla 4. Variables asociadas con la presencia de eventos.

\begin{tabular}{lccccc}
\hline & Eventos & Otros & $p$ & $\begin{array}{c}\text { Multivariado OR } \\
\text { (IC 5\%-95\%) }\end{array}$ & $p$ \\
\hline Sexo (masculino) & $64 \%$ & $59 \%$ & ns & - & 0,046 \\
Edad & $50 \pm 21$ & $57 \pm 20$ & 0,038 & $0,98(0,96-1,0)$ & - \\
SAPS II & $39 \pm 19$ & $34 \pm 25$ & $\mathrm{~ns}$ & - & $\mathrm{ns}$ \\
ARM & $78 \%$ & $49 \%$ & $<0,001$ & $0,62(0,16-2,4)$ & 0,039 \\
Sedoanalgesia & $85 \%$ & $51 \%$ & $<0,001$ & $4,65(1,1-20)$ & $\mathrm{ns}$ \\
Vasopresores & $54 \%$ & $31 \%$ & 0,004 & $1,37(0,6-3.1)$ & 0,001 \\
Estancia en UCl & $14 \pm 13$ & $5 \pm 7$ & $<0,001$ & $1,08(1,03-1,13)$ & - \\
Mortalidad & $29 \%$ & $28 \%$ & 0,96 & & - \\
\hline
\end{tabular}

El reporte voluntario detectó 47 eventos $(43,9 \%)$. Los eventos fueron reportados por médicos residentes en $71 \%$, licenciadas de Enfermería en $22 \%$ y por el médico intensivista en 7\%. De acuerdo con los datos de los reportes, los eventos se produjeron $35,6 \%$ en el turno matutino, en la tarde $31 \%$, en el turno vespertino $9 \%$ y en la noche $24,4 \%$.

La auditoría demostró ser el método más efectivo para la detección de estos eventos, exceptuando los eventos asociados a MAR y el extravío de muestras biológicas. La comparación entre la capacidad de detección de ambos métodos para cada evento en particular se evidencia en la tabla 3.

\section{Presencia de daño y su severidad}

De los 107 eventos detectados, 12,2\% ( $\mathrm{n}=13)$ constituyeron episodios de riesgo (categoría A) y $11,2 \%(n=12)$ incidentes de seguridad (categorías B y C). Éstos se refirieron a la hora del alta de UCI, caídas sin lesiones e incidentes vinculados a MAR, de los cuales cinco constituyeron episodios de riesgo dado que fueron detectados precozmente (tres vinculados a ilegibilidad de la indicación y dos a mala preparación de una solución), y tres incidentes de seguridad que alcanzaron al paciente sin generar daño. Además, incluye las extubaciones no programadas bien toleradas $(\mathrm{n}=3)$.

El restante 76,6\% $(n=82)$ generaron daño de diversa severidad, por lo que se definen como eventos adversos.

En cuanto al daño producido, el $75,6 \%$ de los eventos adversos $(\mathrm{n}=62)$ generó daño transitorio con la necesidad de alguna intervención terapéutica (categoría E). Éstas consistieron en recolocación de sondas, catéteres y drenajes, reintubaciones, colocación de drenajes pleurales, administración de soluciones glucosadas para corrección de hipoglicemias. La prolongación de la estancia en UCI (categoría F) fue producida por el $24,4 \%$ $(n=20)$ de los eventos, de los cuales 13 (15,8\% del total) se debieron a retrasos diagnósticos secundarios a extravío de muestras biológicas, retrasos en estudios imagenológicos de urgencia y en la realización de traqueostomía quirúrgica.

En cuanto a los eventos vinculados a MAR, se detectaron cinco eventos adversos (cuatro hipoglicemias en pacientes con administración de insulina, una hemorragia digestiva en paciente con heparina).

En el período de estudio analizado no se registraron secuelas graves, ni muertes adjudicables a eventos adversos (categorías G, $\mathrm{He}$ e I).

En la figura 2 se muestra la frecuencia según severidad de los eventos clasificadas con el índice NCC MERP.

\section{Variables asociadas a la presencia de eventos vinculados a la seguridad}

Los eventos se presentaron en pacientes con una edad superior con mayor requerimiento de AVM, sedoanalgesia y vasopresores. No se encontraron diferencias significativas en cuanto a su distribución por sexo y no se asoció a mayor severidad de la patología al ingreso valorada por SAPS II. La presencia de eventos vinculados a la seguridad no se asoció a mayor mortalidad $(\mathrm{p}=1)$.

En el análisis multivariado la edad $(\mathrm{p}=0,046)$, el requerimiento de sedoanalgesia $(\mathrm{p}=0,039)$ y los días de estancia en UCI $(p=0,001)$ fueron las variables que se asociaron en forma independiente con la presencia de estos eventos. Los resultados se muestran en la tabla 4.

$\mathrm{Al}$ analizar estas variables, según el número de eventos que presentan, se observó que a mayor número de eventos el requerimiento de AVM, vasopresores y sedoanalgesia, así como la estadía en la unidad, fue superior. Estos resultados se muestran en la tabla 5.

$\mathrm{Si}$ analizamos en forma independiente los eventos más frecuentes, vemos que la salida de sondas, catéteres y drenajes se asoció con el requerimiento de sedoanalge- 
Tabla 5. Asociación entre requerimiento de soporte vital y número de eventos.

\begin{tabular}{lcccc}
\hline & $1(39)$ & $2(10)$ & $>2(12)$ & $p$ \\
\hline Vasopresores & $51 \%$ & $60 \%$ & $67 \%$ & 0,008 \\
ARM & $72 \%$ & $100 \%$ & $83 \%$ & $<0,001$ \\
Sedoanalgesia & $79 \%$ & $100 \%$ & $83 \%$ & $<0,001$ \\
Mortalidad & $31 \%$ & $21 \%$ & $33 \%$ & 0,90 \\
\hline
\end{tabular}

sia $(11 \%$ vs $55 \%$; $=0,026)$ y ARM $(15 \%$ vs $58 \%$; $\mathrm{p}=0,011)$, pero no al de inotrópicos ( $48 \%$ en ambos grupos; $\mathrm{p}=1$ ), así como tampoco a estancias en UCI más prolongadas $(11,5 \pm 10$ vs $16,6 \pm 15$ días; $p=0,13)$. Los eventos asociados a MAR no se asociaron a la estancia en UCI ( $12 \pm 10$ vs $16 \pm 13$ días; $p=0,14)$, así como tampoco al requerimiento de sedoanalgesia ( $22 \%$ vs $21 \%$; $\mathrm{p}=1)$, soporte ventilatorio $(15 \%$ vs $23 \% ; \mathrm{p}=0,7)$ o inotrópico $(11,5 \%$ vs $29 \%$; $\mathrm{p}=0,19)$. Por otro lado, el desarrollo de úlceras por presión (UPP) no se asoció al requerimiento de sedoanalgesia ( $11 \%$ vs $19 \%$; $=1)$, ARM ( $15 \%$ vs $22 \%$; $=1)$, ni inotrópico ( $11,5 \%$ vs $23 \%$; $\mathrm{p}=0,3)$, asociándose a estancia en UCI más prolongada $(10 \pm 7,2$ vs $25,5 \pm 15$ días; $p=0,009)$.

\section{Discusión}

La gravedad de los pacientes ingresados, las barreras de comunicación (entre ellas, el requerimiento de sedación), la realización de un número elevado de actividades por paciente al día ${ }^{(7)}$, y la cantidad y complejidad de la información recibida, entre otros, convierten a las UCI en áreas de riesgo para la aparición de eventos adversos.

La incidencia de EVS en la UCI es elevada, afectando a $35 \%$ de nuestros pacientes. Múltiples estudios epidemiológicos ${ }^{(1,8,9)}$ muestran una tasa de incidencia de entre $4 \%$ y $10 \%$. Estos estudios incluyen a todos los pacientes hospitalizados, constituyendo los de la UCI una mínima proporción de la población. Sin embargo, los pocos pacientes críticos incluidos presentaron una incidencia mayor de incidentes vinculados a la seguridad que los pacientes hospitalizados en otras áreas. El estudio de esta índole de mayor peso publicado hasta el momento es el SYREC ${ }^{(10)}$, que incluyó 1.017 pacientes de los cuales el $58 \%$ presentó al menos un incidente.

Estas series publicadas con grandes poblaciones presentan la característica que utilizaron como método de screening el reporte voluntario por parte de los diferentes miembros del equipo de salud. Esto significa que dada la comprobada existencia de subreporte de eventos, la incidencia real se encuentra subestimada.
Por otro lado, se han publicado estudios que utilizaron la observación directa reportando una incidencia de eventos adversos de entre $20 \%$ y $46 \%{ }^{(11-13)}$.

La auditoría de historias clínicas, que se considera hasta el momento el método gold standard para la evaluación de la incidencia de eventos adversos, es el menos utilizado dado que requiere una gran disponibilidad de recursos humanos. Por otro lado, su fiabilidad depende de la calidad del registro por parte del personal de salud. El mayor trabajo utilizando este método revisó 176 historias clínicas, un número similar al evaluado por nuestro grupo, encontrando una incidencia de $48 \%$ de eventos adversos ${ }^{(14)}$.

Como fortaleza metodológica de nuestro estudio destacamos la utilización de dos metodologías para la detección de eventos vinculados a la seguridad. Mediante ésta se disminuye el número de eventos no detectados. Además, se demostró que ambos métodos son complementarios para la detección de dichos eventos. En nuestra serie se detectó mediante la auditoría el 75\% del total de eventos, lo cual determinaría un sesgo importante si se utilizara este método en forma individual.

En nuestro trabajo los eventos más frecuentes fueron la salida de sondas, catéteres y drenajes $(42,2 \%$ del total), seguidos por los eventos relacionados a MAR (12,8\% del total). La mayoría de los estudios coinciden en que estos dos eventos son los más frecuentes en la UCI. En nuestra investigación buscamos detectar los eventos vinculados a MAR, como fue definida, y no los asociados a medicación en general, lo cual puede explicar la menor incidencia de éstos en relación con las anteriores publicaciones.

De los 107 eventos detectados, el 76,6\% generó algún tipo de daño, constituyendo por lo tanto eventos adversos. La mayor parte $(75,6 \%)$ produjo la prolongación de la estancia en UCI. En el 2006 se publicaron los resultados de la implementación del ICU Incident Safety Reporting System, que evidenció que $40 \%$ de los eventos produjo daño y el $0,8 \%$ contribuyó a la muerte del paciente $^{(15-17)}$. En el SYREC ${ }^{(13)}$, el 34\% de los eventos detectados constituyeron eventos adversos, de los cuales el $29,5 \%$ generó daño temporal y $4,3 \%$ daño permanente, 
comprometió la vida del paciente o contribuyó a su fallecimiento. En el período estudiado en nuestra serie no se detectaron eventos con lesiones graves o causales de fallecimientos. Los estudios coinciden en que la mayoría de los incidentes no generan daños al paciente ${ }^{(18,19)}$.

La mayor parte de los eventos detectados fueron reportados por médicos residentes (71\%) y en segunda instancia por licenciadas de Enfermería (22\%) y médicos intensivistas (7\%). Esto va en contra de anteriores publicaciones, donde Enfermería reporta la mayor cantidad de eventos (60\% en el SYREC). Sin embargo, se observó que estos reportaban principalmente incidentes de seguridad, mientras que los médicos reportaban eventos adversos en mayor proporción ${ }^{(10)}$. Esto último explica, en parte, que en la mayoría de la series se detecta un mayor número de incidentes que de eventos adversos. En nuestra serie, los eventos adversos fueron más frecuentes que los incidentes sin daño, lo cual puede deberse a los eventos seleccionados para el estudio.

Tanto nuestro estudio como el SYREC coinciden en que la mayor parte de los incidentes ocurrieron en el horario de la mañana (36\% vs 52\%). Este hallazgo es compatible con el hecho de que es en dicho turno donde se realiza el mayor número de actividades en los pacientes.

La presencia de EVS se asoció con el tiempo de estancia en UCI cuyo aumento, a su vez, incrementa el número de actividades en el paciente y el riesgo a padecer un incidente. Diferentes estudios demuestran que la posibilidad de que ocurra un eventos adverso se incrementa por día de estancia en UCI entre $8 \%$ y $26 \%{ }^{(20,21)}$. En nuestra serie la salida de sondas, catéteres y drenajes se asoció al requerimiento de ARM, entendible teniendo en cuenta que una proporción importante de estos eventos son extubaciones incidentales y al requerimiento de sedoanalgesia, el cual está vinculado con este último. Por otro lado, el desarrollo de UPP se asoció a estancias en UCI más prolongadas, no relacionándose con el resto de las variables asociadas. Por último, los eventos vinculados a MAR no se asociaron al tiempo de estancia, requerimiento de sedoanalgesia o soporte ventilatorio ni inotrópico, probablemente debido a que son secundarios a descuidos o deficiencias en los procesos vinculados a su prescripción y administración ${ }^{(22,23)}$.

\section{Conclusiones}

- Los eventos vinculados a la seguridad son altamente frecuentes en la UCI.

- La salida de sondas, catéteres y drenajes es el evento más frecuente.

- Su presencia se asocia con el requerimiento de asistencia ventilatoria, vasopresores, sedoanalgesia y la estancia en UCI.
- La auditoría es el método más fiel para la detección de estos eventos.

- El reporte voluntario es una herramienta útil y complementaria al método anterior, siendo más eficaz para la detección de algunos de estos eventos.

\section{Summary}

Introduction: critically ill patients are extremely vulnerable to iatrogenia. Adverse events (AEs) contribute to the morbidity and mortality of hospitalized patients and therefore detection and prevention are of the essence.

Objectives: to study the impact of AEs selected from an intensive care unit and the resulting harm. To set the bases to create a system for the notification of adverse events.

Method: prospective study of impact using two methods: voluntary report and auditing of medical records.

Results: 174 patients were included in the study. 107 adverse events were identified in $35 \%$ of patients. The most prevalent issues were probe and catheter exit-site $(42 \%)$ and those related to high-risk drugs $(12.8 \%)$. Age $(p=0.046)$, length of stay in the ICU $(p=0.001)$ and need of sedoanalgesia $(p=0.039)$ were independently associated to the presence of adverse events. $76.6 \%$ of the events resulted in harm, although they were not associated to higher mortality $(p=0.96)$.

Conclusions: AEs are highly frequent in the intensive care unit. Probe and catheter exit-sites are the most prevalent source. Also, adverse events are related to the need for mechanical ventilation, sedoanalgesia and length of stay in the intensive care unit. Voluntary reporting is a useful tool to identify adverse events.

\section{Resumo}

Introdução: os pacientes em estado crítico são muito vulneráveis à iatrogenia. Os eventos vinculados à segurança (EVS) contribuem para a morbimortalidade dos pacientes internados. Por essa razão sua detecção e prevenção são importantes.

Objetivos: estudar a incidência de EVS selecionados em uma unidade de terapia intensiva (UTI) e os danos que causam. Estabelecer as bases para um sistema de notificação de EVS.

Metodologia: estudo prospectivo de incidência aplicando duas metodologias: relato voluntario e auditoria de prontuários de pacientes.

Resultados: foram incluídos 174 pacientes. Foram detectados 107 EVS em 35\% dos pacientes. Os mais prevalentes foram a saída de sondas e cateteres (42\%) e os relacionados à medicação de alto risco $(12,8 \%)$. A idade $(p=0,046)$, tempo de permanência na UTI 
$(\mathrm{p}=0,001)$ e requerimento de sedoanalgesia $(\mathrm{p}=0,039)$ estão associados independentemente à presença de EVS. 76,6\% dos eventos causou danos, mas não estavam associados a uma maior mortalidade $(\mathrm{p}=0,96)$.

Conclusões: os EVS são muito frequentes na UTI. A saída de sondas e cateteres são os mais prevalentes. Estão associadas ao requerimento de assistência ventilatória, a sedoanalgesia e a permanência na UTI. O relato voluntario é uma ferramenta útil para sua detecção.

\section{Bibliografía}

1. Schimmel EM. The Hazards of Hospitalization. Ann Inter Med 1964; 60:100-10. doi: https://doi.org/10.7326/00034819-60-1-100.

2. Steel K, Gertman PM, Crescenzi C, Anderson J. Iatrogenic illness on a general medical service at a university hospital. N Engl J Med 1981; 304(11):638-42.

3. Leape LL, Brennan TA, Laird N, Lawthers AG, Localio AR, Barnes BA, at al. The nature of adverse events in hospitalized patients. Results of the Harvard Medical Practice Study II. N Engl J Med 1991; 324(6):377-84.

4. Chantler $\mathbf{C}$. The role and education of doctors in the delivery of health care. Lancet 1999; 353(9159):1178-81.

5. Cooper JB, Newbower RS, Long C, McPeek B. Preventable anesthesia mishaps: a study of human factors. Anesthesiology 1978; 49(6):399-406.

6. Hartwig SC, Denger SD, Schneider PJ. Severity-indexed, incident report-based medication error-reporting program. Am J Hosp Pharm 1991; 48(12):2611-6.

7. Donchin Y, Gopher D, Olin M, Badihi Y, Biesky M, Sprung CL, et al. A look into the nature and causes of human errors in the intensive care unit. Crit Care Med 1995; 23(2):294-300.

8. Thomas EJ, Studdert DM, Burstin HR, Orav EJ, Zeena T, Williams EJ, et al. Incidence and types of adverse events and negligent care in Utah and Colorado. Med Care 2000; 38:261-71.

9. Aranaz-Andrés JM, Aibar-Remón C, Vitaller-Murillo J, Ruiz-López P, Limón-Ramírez R, Terol-García E, et al. Incidence of adverse events related to health care in Spain: results of the Spanish National Study of Adverse Events. J Epidemiol Community Health 2008; 62:1022-9.

10. Merino P, Álvarez J, Cruz Martín M, Álonso A, Gutiérrez I; SYREC Study Investigators. Adverse events in Spanish intensive care units: the SYREC study. Int J Qual Health Care 2012; 24(2):105-13.

11. Giraud T, Dhainaut J, Vaxelaire J, Joseph T, Journois D, Bleichner G, et al. Iatrogenic complications in adult intensi- ve care units: a prospective two-center study. Crit Care Med 1993; 21(1):40-51.

12. Bellomo R, Goldsmith D, Russell S, Uchino S. Postoperative serious adverse events in a teaching hospital: a prospective study. Med J Aust 2002; 176(5):216-8

13. Capuzzo M, Nawfal I, Campi M, Valpondi V, Verri M, Alvisi R. Reporting of unintended events in an intensive care unit: comparison between staff and observer. BMC Emerg Med 2005; 5(1):3.

14. Beckmann U, Bohringer C, Carless R, Gillies D, Runciman $\mathbf{W}, \mathbf{W u}$ A, et al. Evaluation of two methods for quality improvement in intensive care: facilitated incident monitoring and retrospective medical chart review. Crit Care Med 2003; 31(4):1006-11.

15. Beckmann U, Baldwin I, Hart G, Runciman W. The Australian Incident Monitoring Study in Intensive Care: AIMS-ICU. An analysis of the first year of reporting. Anaesth Intensive Care 1996; 24(3):320-9.

16. Holzmueller CG, Pronovost PJ, Dickman F, Thompson DA, Wu AW, Lubomski LH, et al. Creating the web-based intensive care unit safety reporting system. J Am Med Inform Assoc 2005; 12(2):130-9.

17. Thomas EJ, Petersen LA. Measuring errors and adverse events in health care. J Gen Intern Med 2003; 18(1):61-7.

18. Graf J, von den Driesch A, Koch KC, Janssens U. Identification and characterization of errors and incidents in a medical intensive care unit. Acta Anaesthesiol Scand 2005; 49(7):930-9.

19. Tissot E, Cornette C, Demoly P, Jacquet M, Barale F, Capellier G. Medication errors at the administration stage in an intensive care unit. Intensive Care Med 1999; 25(4):353-9.

20. Ridley SA, Booth SA, Thompson CM; Intensive Care Society's Working Group on Adverse Incidents. Prescription errors in UK critical care units. Anaesthesia 2004; 59(12):1193-200.

21. Bracco D, Favre JB, Bissonnette B, Wasserfallen JB, Revelly JP, Ravussin P, et al. Human errors in a multidisciplinary intensive care unit: a 1-year prospective study. Intensive Care Med 2001; 27(1):137-45.

22. Flynn EA, Barker KN, Pepper GA, Bates DW, Mikeal RL. Comparison of methods for detecting medication errors in 36 hospitals and skilled-nursing facilities. Am J Health Syst Pharm 2002; 59(5):436-46.

23. Dean B, Barber N. Validity and reliability of observational methods for studying medication administration errors. Am J Health Syst Pharm 2001; 58(1):54-9.

\section{Contribución de autores}

Laureano Leyes, https://orcid.org/0000-0003-2996-5175. Recolección de datos, análisis, redacción y revisión.

Fausto Porcires, https://orcid.org/0000-0002-9708-7792. Diseño, recolección de datos, análisis y revisión.

Mario Godino, https://orcid.org/0000-0003-2413-8605. Diseño, revisión.

Marcelo Barbato, https://orcid.org/0000-0003-4466-4039. Concepción, diseño, análisis, revisión. 